\title{
Upper estimates of transition densities for stable-dominated semigroups
}

\author{
KAMIL KALETA AND PAWEe SZTONYK
}

Abstract. We derive upper estimates of transition densities for Feller semigroups with jump intensities lighter than that of the rotation invariant stable Lévy process.

\section{Introduction and preliminaries}

Let $\alpha \in(0,2)$ and $d=1,2, \ldots$ For the rotation invariant $\alpha$-stable Lévy process on $\mathbb{R}^{d}$ with the Lévy measure

$$
v(\mathrm{~d} y)=\frac{c}{|y|^{\alpha+d}} \mathrm{~d} y, \quad y \in \mathbb{R}^{d} \backslash\{0\},
$$

the asymptotic behavior of its transition densities $p(t, x, y)$ is well known (see, e.g., [2]), i.e.,

$$
p(t, x, y) \approx \min \left(t^{-d / \alpha}, \frac{t}{|y-x|^{\alpha+d}}\right), \quad t>0, x, y \in \mathbb{R}^{d} .
$$

Estimates of densities for more general classes of stable and other jump Lévy processes gradually extended. We would like to mention some of the recent results. Estimates for general stable processes were obtained in [4,32] and for tempered and layered stable processes in [28] and [30]. Other estimates of Lévy and Lévy-type transition densities are discussed by Knopova and Kulik in [21], by Knopova and Schilling in [22] and by Jacob et al. in [20]. Estimates of heat kernels on metric measure spaces having the volume doubling property were obtained by Barlow et al. [1], Chen and Kumagai [7,8], and Grigor'yan et al. [10]. Upper estimates for heat kernels of symmetric jump processes with small jumps of high intensity were obtained by Mimica in [25]. In [24,27], the derivatives of stable densities have been considered, while bounds of heat kernels of the fractional Laplacian perturbed by gradient operators were studied by Bogdan and Jakubowski in [3].

Mathematics Subject Classification (2000): Primary 60J75, 60J35; Secondary 47D03

Keywords: Feller semigroup, heat kernel, transition density, stable-dominated semigroup.

K. Kaleta was supported by the National Science Center (Poland) internship grant on the basis of the decision No. DEC-2012/04/S/ST1/00093. P. Sztonyk was supported by the National Science Center (Poland) grant on the basis of the decision No. DEC-2012/07/B/ST1/03356. 
In [29], estimates of semigroups of stable-dominated Feller operators are given. The corresponding Markov process is a Feller process and not necessarily a Lévy process. The name stable-dominated refers to the fact that the intensity of jumps for the investigated semigroup is dominated by (1). In the present paper, we extend the results obtained in [29] and give estimates from above for a wider class of semigroups with the intensity of jumps lighter than stable processes. We will now describe our results.

Let $f: \mathbb{R}^{d} \times \mathbb{R}^{d} \mapsto[0, \infty]$ be a Borel function. We consider the following assumptions on $f$.

(A.1) There exists a constant $M>0$ such that

$$
f(x, y) \leq M \frac{\phi(|y-x|)}{|y-x|^{\alpha+d}}, \quad x, y \in \mathbb{R}^{d}, y \neq x,
$$

where $\phi:[0, \infty) \rightarrow(0,1]$ is a Borel measurable function such that

(a) $\phi(a)=1$ for $a \in[0,1]$ and there is a constant $c_{1}=c_{1}(\phi)$ such that

$$
\phi(a) \leq c_{1} \phi(b), \quad|a-b| \leq 1,
$$

(b) $\phi \in C^{2}(1, \infty)$ and there is a constant $c_{2}=c_{2}(\phi, \alpha, d)$ such that

$$
\max \left(\left|\phi^{\prime}(a)\right|,\left|\phi^{\prime \prime}(a)\right|\right) \leq c_{2} \phi(a)
$$

for every $a>1$.

(c) there is $c_{3}=c_{3}(\phi, \alpha, d)$ such that

$$
\int_{|x-z| \geq 1,|y-z| \geq 1} \frac{\phi(|y-z|)}{|y-z|^{\alpha+d}} \frac{\phi(|z-x|)}{|z-x|^{\alpha+d}} \mathrm{~d} z \leq c_{3} \frac{\phi(|y-x|)}{|y-x|^{\alpha+d}},
$$

for every $|x-y|>2$.

(A.2) $f(x, x+h)=f(x, x-h)$ for all $x, h \in \mathbb{R}^{d}$ if $\alpha \geq 1$.

(A.3) $f(x, y)=f(y, x)$ for all $x, y \in \mathbb{R}^{d}$.

(A.4) There exists a constant $c_{4}=c_{4}(\phi, \alpha, d)$ such that

$$
\inf _{x \in \mathbb{R}^{d}} \int_{|y-x|>\varepsilon} \frac{f(x, y)}{\phi(|y-x|)} \mathrm{d} y \geq c_{4} \varepsilon^{-\alpha}, \quad \varepsilon>0 .
$$

Denote

$$
b_{\varepsilon}(x)=\int_{|y-x|>\varepsilon} f(x, y) \mathrm{d} y, \quad \varepsilon>0, x \in \mathbb{R}^{d} .
$$

It follows from (A.1) that there is also the constant $c_{5}=c_{5}(\phi, M, \alpha, d)$ such that

$$
\bar{b}_{\varepsilon}:=\sup _{x \in \mathbb{R}^{d}} b_{\varepsilon}(x) \leq c_{5} \varepsilon^{-\alpha}, \quad 0<\varepsilon \leq 1 .
$$

Thus, (A.4) is a partial converse of (A.1) and we have

$$
\underline{b}_{\varepsilon}:=\inf _{x \in \mathbb{R}^{d}} b_{\varepsilon}(x) \geq c_{6} \varepsilon^{-\alpha}, \quad 0<\varepsilon \leq \varepsilon_{0},
$$


for constants $\varepsilon_{0}=\varepsilon_{0}(\phi, M, \alpha, d), c_{6}=c_{6}(\phi, M, \alpha, d)$, since

$$
\begin{aligned}
b_{\varepsilon}(x) & =\int_{|y-x|>\varepsilon} \frac{f(x, y)}{\phi(|y-x|)} \mathrm{d} y-\int_{|y-x|>1} \frac{f(x, y)}{\phi(|y-x|)}(1-\phi(|y-x|)) \mathrm{d} y \\
& \geq c_{4} \varepsilon^{-\alpha}-M \int_{|y-x|>1}|y-x|^{-\alpha-d} \mathrm{~d} y \\
& =c_{4} \varepsilon^{-\alpha}-M c(\alpha, d) \geq c_{6} \varepsilon^{-\alpha}
\end{aligned}
$$

provided $\varepsilon^{\alpha} \leq \frac{c_{4}-c_{6}}{M c(\alpha, d)}$.

We note that the assumption (A.1)(c) is satisfied for every nonincreasing function $\phi:(0, \infty) \rightarrow(0,1]$ such that

$$
\phi(a) \phi(b) \leq c \phi(a+b), \quad a, b>1,
$$

for some positive constant $c$. Therefore, it is easy to verify that all the assumptions on $\phi$ are satisfied, e.g., for functions $\phi(s)=\mathrm{e}^{\left(1-s^{\beta}\right)} \wedge 1$, where $\beta \in(0,1], \phi(s)=(1 \vee s)^{-\gamma}$, where $\gamma>0, \phi(s)=1 / \log (e(s \vee 1)), \phi(s)=1 / \log \log \left(e^{e}(s \vee 1)\right)$, and all their products and positive powers.

It is also reasonable to ask whether the conditions in the assumption (A.1) are satisfied by more general functions of the form

$$
\phi(s)= \begin{cases}1 & \text { if } s \in[0,1], \\ \mathrm{e}^{-m s^{\beta}} s^{\gamma} & \text { if } s>1, \text { with } m, \beta>0, \gamma \in \mathbb{R} .\end{cases}
$$

In this case, both conditions (a) and (b) on $\phi$ hold for $\beta \in(0,1]$ with no further restrictions on parameters $m$ and $\gamma$, while, as proven in Sect. 3, the condition (c) is satisfied when $\beta \in(0,1]$ and $\gamma<d / 2+\alpha-1 / 2$. Furthermore, this restriction on parameters is essential (see Remark 1 in Section 3). Note also that this range of $\beta$ and $\gamma$ in (2) covers, e.g., jump intensities dominated by those of isotropic relativistic stable processes (see, e.g., [23, Lemma 2.3]).

For $x \in \mathbb{R}^{d}$ and $r>0$, we let $B(x, r)=\left\{y \in \mathbb{R}^{d}:|y-x|<r\right\} . B_{b}\left(\mathbb{R}^{d}\right)$ denotes the set of bounded Borel measurable functions, $C_{c}^{k}\left(\mathbb{R}^{d}\right)$ denotes the set of $k$ times continuously differentiable functions with compact support, and $C_{\infty}\left(\mathbb{R}^{d}\right)$ is the set of continuous functions vanishing at infinity. We use $c, C$ (with subscripts) to denote finite positive constants which depend only on $\phi, M, \alpha$, and the dimension $d$. Any additional dependence is explicitly indicated by writing, e.g., $c=c(n)$. The value of $c, C$, when used without subscripts, may change from place to place. We write $f(x) \approx g(x)$ to indicate that there is a constant $c$ such that $c^{-1} f(x) \leq g(x) \leq c f(x)$.

Under the assumptions (A.1) and (A.2), we may consider the operator

$$
\begin{aligned}
\mathcal{A} \varphi(x)= & \lim _{\varepsilon \downarrow 0} \int_{|y-x|>\varepsilon}(\varphi(y)-\varphi(x)) f(x, y) \mathrm{d} y \\
= & \int_{\mathbb{R}^{d}}\left(\varphi(x+h)-\varphi(x)-h \cdot \nabla \varphi(x) \mathbf{1}_{|h|<1}\right) f(x, x+h) \mathrm{d} h \\
& +\frac{1}{2} \int_{|h|<1} h \cdot \nabla \varphi(x)(f(x, x+h)-f(x, x-h)) \mathrm{d} h, \quad \varphi \in C_{c}^{2}\left(\mathbb{R}^{d}\right) .
\end{aligned}
$$


Recall the following basic fact (see [29, Lemma 1]).

LEMMA 1. If (A.1) and (A.2) hold and the function $x \rightarrow f(x, y)$ is continuous on $\mathbb{R}^{d} \backslash\{y\}$ for every $y \in \mathbb{R}^{d}$, then $\mathcal{A}$ maps $C_{c}^{2}\left(\mathbb{R}^{d}\right)$ into $C_{\infty}\left(\mathbb{R}^{d}\right)$.

In the following, we always assume that the condition (A.1) is satisfied. For every $\varepsilon>0$, we denote

$$
f_{\varepsilon}(x, y)=\mathbf{1}_{B(0, \varepsilon)^{c}}(y-x) f(x, y), \quad x, y \in \mathbb{R}^{d},
$$

and

$$
\mathcal{A}_{\varepsilon} \varphi(x)=\int(\varphi(y)-\varphi(x)) f_{\varepsilon}(x, y) \mathrm{d} y, \quad \varphi \in B_{b}\left(\mathbb{R}^{d}\right) .
$$

Note that the operators $\mathcal{A}_{\varepsilon}$ are bounded since $\left|\mathcal{A}_{\varepsilon} \varphi(x)\right| \leq 2\|\varphi\|_{\infty} b_{\varepsilon}(x) \leq$ $2 \bar{b}_{\varepsilon}\|\varphi\|_{\infty}$. Therefore, the operator

$$
\mathrm{e}^{t \mathcal{A}_{\varepsilon}}=\sum_{n=0}^{\infty} \frac{t^{n} \mathcal{A}_{\varepsilon}^{n}}{n !}, \quad t \geq 0, \varepsilon>0
$$

is well defined and bounded from $B_{b}\left(\mathbb{R}^{d}\right)$ to $B_{b}\left(\mathbb{R}^{d}\right)$. In fact, for every $\varepsilon>0$, the family of operators $\left\{\mathrm{e}^{t \mathcal{A}_{\varepsilon}}, t \leq 0\right\}$ is a semigroup on $B_{b}\left(\mathbb{R}^{d}\right)$, i.e., $\mathrm{e}^{(t+s) \mathcal{A}_{\varepsilon}}=\mathrm{e}^{t \mathcal{A}_{\varepsilon}} \mathrm{e}^{s \mathcal{A}_{\varepsilon}}$ for all $t, s \geq 0, \varphi \in B_{b}\left(\mathbb{R}^{d}\right)$. We note that $\mathrm{e}^{t \mathcal{A}_{\varepsilon}}$ is positive for all $t \geq 0, \varepsilon>0$ (see (5)).

Our first result is the following theorem.

THEOREM 1. If (A.1)-(A.4) are satisfied, then there exists the constants $C_{1}$ and $C_{2}$ such that for every nonnegative $\varphi \in B_{b}\left(\mathbb{R}^{d}\right)$ and $\varepsilon \in\left(0, \varepsilon_{0} \wedge 1\right)$, we have

$$
\mathrm{e}^{t \mathcal{A}_{\varepsilon}} \varphi(x) \leq C_{1} \mathrm{e}^{C_{2} t} \int \varphi(y) \min \left(t^{-d / \alpha}, \frac{t \phi(|y-x|)}{|y-x|^{\alpha+d}}\right) \mathrm{d} y+\mathrm{e}^{-t b_{\varepsilon}(x)} \varphi(x),
$$

for every $x \in \mathbb{R}^{d}$.

The proof of Theorem 1 is given in Sect. 2. To study a limiting semigroup, we will need some additional assumptions.

(A.5) The function $x \rightarrow f(x, y)$ is continuous on $\mathbb{R}^{d} \backslash\{y\}$ for every $y \in \mathbb{R}^{d}$.

(A.6) $\mathcal{A}$ regarded as an operator on $C_{\infty}\left(\mathbb{R}^{d}\right)$ is closable and its closure $\overline{\mathcal{A}}$ is a generator of a strongly continuous contraction semigroup of operators $\left\{P_{t}, t \geq 0\right\}$ on $C_{\infty}\left(\mathbb{R}^{d}\right)$.

Clearly, for every $\varphi \in C_{c}^{2}\left(\mathbb{R}^{d}\right)$ with $\sup _{x \in \mathbb{R}^{d}} \varphi(x)=\varphi\left(x_{0}\right) \geq 0$, we have $\mathcal{A} \varphi\left(x_{0}\right) \leq$ 0 , i.e., $\mathcal{A}$ satisfies the positive maximum principle. This implies that all $P_{t}(t \geq 0)$ are positive operators (see [9, Theorems 1.2.12 and 4.2.2]). Thus, by our assumptions, $\left\{P_{t}, t \geq 0\right\}$ is a Feller semigroup.

The following theorem is our main result. 
THEOREM 2. If (A.1)-(A.6) hold, then there is $p:(0, \infty) \times \mathbb{R}^{d} \times \mathbb{R}^{d} \rightarrow[0, \infty)$ such that

$$
P_{t} \varphi(x)=\int_{\mathbb{R}^{d}} \varphi(y) p(t, x, y) d y, \quad x \in \mathbb{R}^{d}, t>0, \varphi \in C_{\infty}\left(\mathbb{R}^{d}\right),
$$

and

$$
p(t, x, y) \leq C_{1} \mathrm{e}^{C_{2} t} \min \left(t^{-d / \alpha}, \frac{t \phi(|y-x|)}{|y-x|^{\alpha+d}}\right), \quad x, y \in \mathbb{R}^{d}, t>0 .
$$

We note that $\mathcal{A}$ is conservative, i.e., for $\varphi \in C_{c}^{\infty}\left(\mathbb{R}^{d}\right)$ such that $0 \leq \varphi \leq 1, \varphi(0)=1$, and $\varphi_{k}(x)=\varphi(x / k)$, we have $\sup _{k \in \mathbb{N}}\left\|\mathcal{A} \varphi_{k}\right\|_{\infty}<\infty$, and $\lim _{k \rightarrow \infty}\left(\mathcal{A} \varphi_{k}\right)(x)=0$, for every $x \in \mathbb{R}^{d}$. It follows from Theorem 4.2.7 in [9] that there exists a Markov process $\left\{X_{t}, t \geq 0\right\}$ such that $\mathbb{E}\left[\varphi\left(X_{t}\right) \mid X_{0}=x\right]=P_{t} \varphi(x)$.

It is known that every generator $G$ of a Feller semigroup with $C_{c}^{\infty}\left(\mathbb{R}^{d}\right) \subset \mathcal{D}(G)$ is necessarily of the form

$$
\begin{aligned}
G \varphi(x)= & \sum_{i, j=1}^{d} q_{i j}(x) D_{x_{i}} D_{x_{j}} \varphi(x)+l(x) \nabla \varphi(x)-c(x) \varphi(x) \\
& +\int_{\mathbb{R}^{d}}\left(\varphi(x+h)-\varphi(x)-h \cdot \nabla \varphi(x) \mathbf{1}_{|h|<1}\right) v(x, \mathrm{~d} h),
\end{aligned}
$$

where $\varphi \in C_{c}^{\infty}\left(\mathbb{R}^{d}\right), q(x)=\left(q_{i j}(x)\right)_{i, j=1}^{n}$ is a nonnegative definite real symmetric matrix, the vector $l(x)=\left(l_{i}(x)\right)_{i=1}^{d}$ has real coordinates, $c(x) \geq 0$, and $v(x, \cdot)$ is a Lévy measure (see [17, Chapter 4.5]).

The converse problem whether a given operator $G$ generates a Feller semigroup is not completely resolved yet. For the interested reader, we remark that criteria are given, e.g., in [13-16,18]. Generally, smoothness of the coefficients $q, l, c, v$ in (4) is sufficient for the existence (see Theorem 5.24 in [12], Theorem 4.6.7 in [19] and Lemma 2 in [29]). Other conditions are given also in [26].

Chen et al. [6] and Chen and Kumagai [7,8] investigate the case of symmetric jumptype Markov processes on metric measure spaces by using Dirichlet forms. Under the assumption that the corresponding jump kernels are comparable with certain rotation invariant functions, they prove the existence and obtain estimates of the densities (see Theorem 1.2 in [6]) analogous to (3). In the present paper, we propose completely different approach which is based on general approximation scheme recently devised in [29]. In Theorem 2, we assume the estimate (A.1) from above but we use (A.4) as the only estimate for the size of $f$ from below. We also emphasize that we obtain exactly $\phi(|x-y|)$ in (3) and from [8,6] follow estimates with $\phi(c|x-y|)$ for some constant $c \in(0,1)$. This seems to be essential especially in the case of exponentially localized Lévy measures. Our general framework, including a layout of lemmas, is similar to that in [29]. However, in the present case, the decay of the jump intensity may be significantly lighter than stable, and therefore, much more subtle argument is needed. Note that the new condition (A.1)(c), which is pivotal for our further investigations, is necessary for the two-sided sharp bounds similar to the right-hand side of (3). 


\section{Approximation}

In this section, we apply an approximation scheme recently devised in [29] (we note that an alternative approximation scheme is given in [5]). We recall that

$$
f_{\varepsilon}(x, y)=\mathbf{1}_{B(0, \varepsilon)^{c}}(y-x) f(x, y), \quad \varepsilon>0, x, y \in \mathbb{R}^{d},
$$

and

$$
b_{\varepsilon}(x)=\int_{|y-x|>\varepsilon} f(x, y) \mathrm{d} y=\int f_{\varepsilon}(x, y) \mathrm{d} y, \quad \varepsilon>0, x \in \mathbb{R}^{d} .
$$

We have

$$
\begin{aligned}
\mathcal{A}_{\varepsilon} \varphi(x) & =\int(\varphi(y)-\varphi(x)) f_{\varepsilon}(x, y) \mathrm{d} y+\left(\bar{b}_{\varepsilon}-b_{\varepsilon}(x)\right) \int(\varphi(y)-\varphi(x)) \delta_{x}(\mathrm{~d} y) \\
& =\int(\varphi(y)-\varphi(x)) \tilde{\nu}_{\varepsilon}(x, \mathrm{~d} y) \\
& =\Gamma_{\varepsilon} \varphi(x)-\bar{b}_{\varepsilon} \varphi(x), \quad \varphi \in B_{b}\left(\mathbb{R}^{d}\right), x \in \mathbb{R}^{d},
\end{aligned}
$$

where

$$
\tilde{v}_{\varepsilon}(x, \mathrm{~d} y)=f_{\varepsilon}(x, y) \mathrm{d} y+\left(\bar{b}_{\varepsilon}-b_{\varepsilon}(x)\right) \delta_{x}(\mathrm{~d} y),
$$

and

$$
\Gamma_{\varepsilon} \varphi(x)=\int \varphi(y) \tilde{v}_{\varepsilon}(x, \mathrm{~d} y), \quad \varphi \in B_{b}\left(\mathbb{R}^{d}\right), x \in \mathbb{R}^{d} .
$$

This yields that

$$
\mathrm{e}^{t \mathcal{A}_{\varepsilon}} \varphi(x)=\mathrm{e}^{t\left(\Gamma_{\varepsilon}-\bar{b}_{\varepsilon} I\right)} \varphi(x)=\mathrm{e}^{-t \bar{b}_{\varepsilon}} \mathrm{e}^{t \Gamma_{\varepsilon}} \varphi(x) .
$$

A consequence of (5) is that we may consider the operator $\Gamma_{\varepsilon}$ and its powers instead of $\mathcal{A}_{\varepsilon}$. The fact that $\Gamma_{\varepsilon}$ is positive enables for more precise estimates.

For $n \in \mathbb{N}$, we define

$$
\begin{aligned}
f_{n+1, \varepsilon}(x, y)= & \int f_{n, \varepsilon}(x, z) f_{\varepsilon}(z, y) \mathrm{d} z \\
& +\left(\bar{b}_{\varepsilon}-b_{\varepsilon}(y)\right) f_{n, \varepsilon}(x, y)+\left(\bar{b}_{\varepsilon}-b_{\varepsilon}(x)\right)^{n} f_{\varepsilon}(x, y),
\end{aligned}
$$

where we let $f_{1, \varepsilon}=f_{\varepsilon}$. By induction and Fubini-Tonelli theorem, we get

$$
\int f_{n, \varepsilon}(x, y) \mathrm{d} y=\bar{b}_{\varepsilon}^{n}-\left(\bar{b}_{\varepsilon}-b_{\varepsilon}(x)\right)^{n}, \quad x \in \mathbb{R}^{d}, n \in \mathbb{N} .
$$

Also, it was proved in [29, Lemma 3] that for all $\varepsilon>0, x \in \mathbb{R}^{d}$, and $n \in \mathbb{N}$

$$
\Gamma_{\varepsilon}^{n} \varphi(x)=\int \varphi(z) f_{n, \varepsilon}(x, z) \mathrm{d} z+\left(\bar{b}_{\varepsilon}-b_{\varepsilon}(x)\right)^{n} \varphi(x),
$$

whenever $\varphi \in B_{b}\left(\mathbb{R}^{d}\right)$.

The next lemma is crucial for our further investigation. It is essential that we obtain precisely the constants equal to one before $b_{\varepsilon}(y)$ below. 
LEMMA 2. We have the following.

(1) If (A.1), (A.2) and (A.4) hold then there is a constant $c_{7}=c_{7}(\phi, M, \alpha, d)$ and the number $\kappa \in(0,1)$ such that

$$
\int_{B(y, \kappa|y-x|)}|z-x|^{-\alpha-d} f_{\varepsilon}(y, z) \mathrm{d} z \leq\left(b_{\varepsilon}(y)+c_{7}\right)|y-x|^{-\alpha-d},
$$

for every $\varepsilon \in(0,1)$ and for every $x, y \in \mathbb{R}^{d}$.

(2) If (A.1) and (A.2) hold then there is a constant $c_{8}=c_{8}(\phi, M, \alpha, d)$ such that

$$
\int_{B(y, 1)} \frac{\phi(|z-x|)}{|z-x|^{\alpha+d}} f_{\varepsilon}(y, z) \mathrm{d} z \leq\left(b_{\varepsilon}(y)+c_{8}\right) \frac{\phi(|y-x|)}{|y-x|^{\alpha+d}},
$$

for every $\varepsilon \in(0,1)$ and for every $|x-y|>2$.

Proof. First, we prove the statement (1). We have

$$
\begin{aligned}
& \int_{B(y, \kappa|y-x|)}|z-x|^{-\alpha-d} f_{\varepsilon}(y, z) \mathrm{d} z \\
& =\int_{B(y, \kappa|y-x|)}\left[|z-x|^{-\alpha-d}-|y-x|^{-\alpha-d}\right] f_{\varepsilon}(y, z) \mathrm{d} z \\
& \quad+|y-x|^{-\alpha-d} \int_{B(y, \kappa|y-x|)} f_{\varepsilon}(y, z) \mathrm{d} z .
\end{aligned}
$$

We only need to estimate the first integral on the right-hand side of the above equality. Denote $\theta(z):=|z-x|^{-\alpha-d},|z-x|>0$.

$$
\partial_{j} \theta(z)=(\alpha+d)|z-x|^{-\alpha-d-2}\left(x_{j}-z_{j}\right),
$$

and

$$
\partial_{j, k} \theta(z)=(\alpha+d)|z-x|^{-\alpha-d-2}\left[(\alpha+d+2) \frac{\left(x_{j}-z_{j}\right)\left(x_{k}-z_{k}\right)}{|x-z|^{2}}-\delta_{j k}\right] .
$$

This yields

$$
\sup _{\substack{z \in B(y, \kappa|y-x|), j \in\{1, \ldots, d\}}}\left|\partial_{j} \theta(z)\right| \leq(\alpha+d)(1-\kappa)^{-\alpha-d-1}|y-x|^{-\alpha-d-1},
$$

and

$$
\sup _{\substack{z \in B(y, \kappa|y-x|), j, k \in\{1, \ldots, d\}}}\left|\partial_{j, k} \theta(z)\right| \leq(\alpha+d)(\alpha+d+3)(1-\kappa)^{-\alpha-d-2}|y-x|^{-\alpha-d-2},
$$


for every $\kappa \in(0,1)$. We consider now two cases. Let first $\alpha<1$. Using the Taylor expansion for $\theta,(8)$ and (A.1), we get

$$
\begin{aligned}
& \left|\int_{B(y, \kappa|y-x|)}\left[|z-x|^{-\alpha-d}-|y-x|^{-\alpha-d}\right] f_{\varepsilon}(y, z) \mathrm{d} z\right| \\
& \quad=\left|\int_{B(0, \kappa|y-x|)}(\theta(y+h)-\theta(y)) f_{\varepsilon}(y, y+h) \mathrm{d} h\right| \\
& \quad \leq C(1-\kappa)^{-\alpha-d-1}|y-x|^{-\alpha-d-1} \int_{B(0, \kappa|y-x|)}|h| f_{\varepsilon}(y, y+h) \mathrm{d} h \\
& \quad \leq C \kappa^{1-\alpha}(1-\kappa)^{-\alpha-d-1}|y-x|^{-\alpha-d}|y-x|^{-\alpha} .
\end{aligned}
$$

Let now $\alpha \geq 1$. Again, using the Taylor expansion for $\theta$, (9), (A.1) and (A.2), we obtain

$$
\begin{aligned}
& \left|\int_{B(y, \kappa|y-x|)}\left[|z-x|^{-\alpha-d}-|y-x|^{-\alpha-d}\right] f_{\varepsilon}(y, z) \mathrm{d} z\right| \\
& \quad=\left|\int_{B(0, \kappa|y-x|)}(\theta(y+h)-\theta(y)-\nabla \theta(y) \cdot h) f_{\varepsilon}(y, y+h) \mathrm{d} h\right| \\
& \quad \leq C(1-\kappa)^{-\alpha-d-2}|y-x|^{-\alpha-d-2} \int_{B(0, \kappa|y-x|)}|h|^{2} f_{\varepsilon}(y, y+h) \mathrm{d} h \\
& \quad \leq C \kappa^{2-\alpha}(1-\kappa)^{-\alpha-d-2}|y-x|^{-\alpha-d}|y-x|^{-\alpha} .
\end{aligned}
$$

We thus see that by the two above estimates and by (A.4), we finally have

$$
\begin{aligned}
& \left|\int_{B(y, \kappa|y-x|)}\left[|z-x|^{-\alpha-d}-|y-x|^{-\alpha-d}\right] f_{\varepsilon}(y, z) \mathrm{d} z\right| \\
& \quad \leq|y-x|^{-\alpha-d}\left(\mathbf{1}_{\left\{|y-x|<\varepsilon_{0}\right\}} \int_{|z-y|>\kappa|y-x|} f(y, z) \mathrm{d} z+c_{7} \mathbf{1}_{\left\{|y-x| \geq \varepsilon_{0}\right\}}\right),
\end{aligned}
$$

for sufficiently small $\kappa \in(0,1)$. This ends the proof of (1).

We now show the statement (2). Let $|x-y|>2$. Similarly as before, we have

$$
\begin{gathered}
\int_{B(y, 1)} \frac{\phi(|z-x|)}{|z-x|^{\alpha+d}} f_{\varepsilon}(y, z) \mathrm{d} z= \\
\int_{B(y, 1)}\left[\frac{\phi(|z-x|)}{|z-x|^{\alpha+d}}-\frac{\phi(|y-x|)}{|y-x|^{\alpha+d}}\right] f_{\varepsilon}(y, z) \mathrm{d} z \\
+\frac{\phi(|y-x|)}{|y-x|^{\alpha+d}} \int_{B(y, 1)} f_{\varepsilon}(y, z) \mathrm{d} z .
\end{gathered}
$$

Observe that it is enough to estimate the first integral on the right-hand side of the above-displayed equality. Denote $\eta(z):=\phi(|z-x|)|z-x|^{-\alpha-d}$. Clearly, by (A.1) (a)-(b), we have

$$
\max \left(\sup _{\substack{z \in B(y, 1), j \in\{1, \ldots, d\}}}\left|\partial_{j} \eta(z)\right|, \sup _{\substack{z \in B(y, 1), j, k \in\{1, \ldots, d\}}}\left|\partial_{j, k} \eta(z)\right|\right) \leq C \eta(y) .
$$


Using the Taylor expansion for $\eta,(10),($ A.1) and (A.2), we obtain

$$
\begin{aligned}
& \left|\int_{B(y, 1)}\left[\frac{\phi(|z-x|)}{|z-x|^{\alpha+d}}-\frac{\phi(|y-x|)}{|y-x|^{\alpha+d}}\right] f_{\varepsilon}(y, z) \mathrm{d} z\right| \\
& \quad=\left|\int_{B(0,1)}(\eta(y+h)-\eta(y)) f_{\varepsilon}(y, y+h) \mathrm{d} h\right| \\
& \leq\left|\int_{B(0,1)}(\eta(y+h)-\eta(y)-\nabla \eta(y) \cdot h) f_{\varepsilon}(y, y+h) \mathrm{d} h\right| \\
& \quad+\left|\int_{B(0,1)} \nabla \eta(y) \cdot h \frac{f_{\varepsilon}(y, y+h)-f_{\varepsilon}(y, y-h)}{2} \mathrm{~d} h\right| \\
& \leq c_{8} \eta(y) .
\end{aligned}
$$

which ends the proof.

We now obtain the estimates of $f_{n, \varepsilon}(x, y)$. Our argument in the proof of the following lemma shows the significance of assumptions on the dominating function $\phi$.

LEMMA 3. If (A.1)-(A.4) hold then:

(1) there exists a constant $c_{9}=c_{9}(\phi, M, \alpha, d)$ such that

$$
f_{n, \varepsilon}(x, y) \leq c_{9} n\left(\bar{b}_{\varepsilon}+c_{7}\right)^{n-1}|y-x|^{-\alpha-d},
$$

for every $x, y \in \mathbb{R}^{d}, \varepsilon \in(0,1), n \in \mathbb{N}$,

(2) there exists the constants $c_{10}=c_{10}(\phi, M, \alpha, d)$ and $c_{11}=c_{11}(\phi, M, \alpha, d)$ such that

$$
f_{n, \varepsilon}(x, y) \leq c_{10} n\left(\bar{b}_{\varepsilon}+c_{11}\right)^{n-1} \frac{\phi(|y-x|)}{|y-x|^{\alpha+d}},
$$

for every $x, y \in \mathbb{R}^{d}, \varepsilon \in(0,1), n \in \mathbb{N}$.

Proof. We use induction. Clearly, for $n=1$, both inequalities hold with constants $c_{9}=M, c_{10}=M$ (and an arbitrary positive $c_{11}$ ), respectively. Consider first the inequality in (1). We will prove that it holds with constant $c_{9}=M \kappa^{-\alpha-d}$, where $\kappa \in(0,1)$ is the number from previous lemma.

Let

$$
\int f_{n, \varepsilon}(x, z) f_{\varepsilon}(z, y) \mathrm{d} z=\int_{B(y, \kappa|y-x|)^{c}}+\int_{B(y, \kappa|y-x|)}=I+I I .
$$

By (A.1) (a) and (6), we have

$$
\begin{aligned}
I & \leq \kappa^{-\alpha-d} M|y-x|^{-\alpha-d} \int f_{n, \varepsilon}(x, z) \mathrm{d} z \\
& =\kappa^{-\alpha-d} M|y-x|^{-\alpha-d}\left[\bar{b}_{\varepsilon}^{n}-\left(\bar{b}_{\varepsilon}-b_{\varepsilon}(x)\right)^{n}\right] .
\end{aligned}
$$


By symmetry of $f$ (see (A.3)), induction, and Lemma 2 (1), we also have

$$
\begin{aligned}
I I & \leq c_{9} n\left(\bar{b}_{\varepsilon}+c_{7}\right)^{n-1} \int_{B(y, \kappa|y-x|)}|x-z|^{-\alpha-d} f_{\varepsilon}(y, z) \mathrm{d} z \\
& \leq c_{9} n\left(\bar{b}_{\varepsilon}+c_{7}\right)^{n-1}\left(b_{\varepsilon}(y)+c_{7}\right)|x-y|^{-\alpha-d} .
\end{aligned}
$$

We get

$$
\begin{aligned}
f_{n+1, \varepsilon}(x, y)= & I+I I+\left(\bar{b}_{\varepsilon}-b_{\varepsilon}(y)\right) f_{n, \varepsilon}(x, y)+\left(\bar{b}_{\varepsilon}-b_{\varepsilon}(x)\right)^{n} f_{\varepsilon}(x, y) \\
\leq & M \kappa^{-\alpha-d}\left[\bar{b}_{\varepsilon}^{n}-\left(\bar{b}_{\varepsilon}-b_{\varepsilon}(x)\right)^{n}\right]|y-x|^{-\alpha-d} \\
& +c_{9} n\left(\bar{b}_{\varepsilon}+c_{7}\right)^{n-1}\left(b_{\varepsilon}(y)+c_{7}\right)|x-y|^{-\alpha-d} \\
& +\left(\bar{b}_{\varepsilon}-b_{\varepsilon}(y)\right) c_{9} n\left(\bar{b}_{\varepsilon}+c_{7}\right)^{n-1}|x-y|^{-\alpha-d} \\
& +\left(\bar{b}_{\varepsilon}-b_{\varepsilon}(x)\right)^{n} M|x-y|^{-\alpha-d} \\
\leq & c_{9}(n+1)\left(\bar{b}_{\varepsilon}+c_{7}\right)^{n}|x-y|^{-\alpha-d},
\end{aligned}
$$

which ends the proof of part (1).

We now complete the proof of the inequality in (2). We will prove that it holds with constants $c_{10}=c_{1} \max \left(c_{9}, 2^{\alpha+d} M\right)$ and $c_{11}=\max \left(c_{7}, c_{8}+M c_{3}\right)$. When $|x-y| \leq 2$, then it directly follows from the part (1) and (A.1)(a). Assume now that $|x-y|>2$. We have

$$
\int f_{n, \varepsilon}(x, z) f_{\varepsilon}(z, y) \mathrm{d} z=\int_{B(x, 1)}+\int_{B(x, 1)^{c}}=I+I I .
$$

By (A.1) (a) and (6), we get

$$
\begin{aligned}
I & \leq 2^{\alpha+d} M c_{1} \frac{\phi(|x-y|)}{|y-x|^{\alpha+d}} \int f_{n, \varepsilon}(x, z) \mathrm{d} z \\
& =2^{\alpha+d} M c_{1} \frac{\phi(|x-y|)}{|y-x|^{\alpha+d}}\left[\bar{b}_{\varepsilon}^{n}-\left(\bar{b}_{\varepsilon}-b_{\varepsilon}(x)\right)^{n}\right] .
\end{aligned}
$$

By symmetry of $f$ (see (A.3)), induction, and Lemma 2 (2) and (A.1) (c), we also have

$$
\begin{aligned}
I I & \leq c_{10} n\left(\bar{b}_{\varepsilon}+c_{11}\right)^{n-1} \int_{B(x, 1)^{c}} \frac{\phi(|x-z|)}{|x-z|^{\alpha+d}} f_{\varepsilon}(y, z) \mathrm{d} z \\
& \leq c_{10} n\left(\bar{b}_{\varepsilon}+c_{11}\right)^{n-1}\left(b_{\varepsilon}(y)+c_{8}+M c_{3}\right) \frac{\phi(|x-y|)}{|x-y|^{\alpha+d}} .
\end{aligned}
$$

We get

$$
\begin{aligned}
f_{n+1, \varepsilon}(x, y)= & I+I I+\left(\bar{b}_{\varepsilon}-b_{\varepsilon}(y)\right) f_{n, \varepsilon}(x, y)+\left(\bar{b}_{\varepsilon}-b_{\varepsilon}(x)\right)^{n} f_{\varepsilon}(x, y) \\
\leq & 2^{\alpha+d} M c_{1}\left[\bar{b}_{\varepsilon}^{n}-\left(\bar{b}_{\varepsilon}-b_{\varepsilon}(x)\right)^{n}\right] \frac{\phi(|y-x|)}{|y-x|^{\alpha+d}} \\
& +c_{10} n\left(\bar{b}_{\varepsilon}+c_{11}\right)^{n-1}\left(b_{\varepsilon}(y)+c_{8}+M c_{3}\right) \frac{\phi(|x-y|)}{|x-y|^{\alpha+d}}
\end{aligned}
$$




$$
\begin{aligned}
& +\left(\bar{b}_{\varepsilon}-b_{\varepsilon}(y)\right) c_{10} n\left(\bar{b}_{\varepsilon}+c_{11}\right)^{n-1} \frac{\phi(|x-y|)}{|x-y|^{\alpha+d}} \\
& +\left(\bar{b}_{\varepsilon}-b_{\varepsilon}(x)\right)^{n} M \frac{\phi(|x-y|)}{|x-y|^{\alpha+d}} \\
& \leq c_{10}(n+1)\left(\bar{b}_{\varepsilon}+c_{11}\right)^{n} \frac{\phi(|x-y|)}{|x-y|^{\alpha+d}} .
\end{aligned}
$$

LEMMA 4. Assume (A.1), (A.3), and(A.4). Then, there exists $c_{12}=c_{12}(\phi, M, \alpha, d)$ such that

$$
f_{n, \varepsilon}(x, y) \leq c_{12} \bar{b}_{\varepsilon}^{d / \alpha}\left(\bar{b}_{\varepsilon}^{n}-\left(\bar{b}_{\varepsilon}-b_{\varepsilon}(x)\right)^{n}\right), \quad x, y \in \mathbb{R}^{d}, \varepsilon \in\left(0, \varepsilon_{0}\right), n \in \mathbb{N} .
$$

Proof. For $n=1$ by (A.1) and (A.4), we have

$$
f_{\varepsilon}(x, y) \leq \frac{M}{\varepsilon^{\alpha+d}} \leq M\left(\frac{b_{\varepsilon}(x)}{c_{6}}\right)^{(\alpha+d) / \alpha} \leq M\left(\frac{b_{\varepsilon}(x)}{c_{6}}\right)\left(\frac{\bar{b}_{\varepsilon}}{c_{6}}\right)^{d / \alpha}
$$

and so (11) holds with $c_{12}=M c_{6}{ }^{-d / \alpha-1}$. Let (11) holds for some $n \in \mathbb{N}$ with $c_{12}=M c_{6}{ }^{-d / \alpha-1}$. By induction and the symmetry of $f_{\varepsilon}$, we get

$$
\begin{aligned}
f_{n+1, \varepsilon}(x, y) \leq & c_{12} \bar{b}_{\varepsilon}^{d / \alpha}\left(\bar{b}_{\varepsilon}^{n}-\left(\bar{b}_{\varepsilon}-b_{\varepsilon}(x)\right)^{n}\right)\left(\int f_{\varepsilon}(y, z) \mathrm{d} z+\bar{b}_{\varepsilon}-b_{\varepsilon}(y)\right) \\
& +\left(\bar{b}_{\varepsilon}-b_{\varepsilon}(x)\right)^{n} c_{12} \bar{b}_{\varepsilon}^{d / \alpha} b_{\varepsilon}(x) \\
= & c_{12}\left(\bar{b}_{\varepsilon}\right)^{d / \alpha}\left(\bar{b}_{\varepsilon}^{n+1}-\left(\bar{b}_{\varepsilon}-b_{\varepsilon}(x)\right)^{n+1}\right) .
\end{aligned}
$$

In the following lemma, we will need some additional notations. For a function $g$, we denote $b_{\varepsilon}^{g}(x):=\int_{|y-x|>\varepsilon} g(|y-x|) f_{\varepsilon}(x, y) \mathrm{d} y$ and $\bar{b}_{\varepsilon}^{g}=\sup _{x \in \mathbb{R}^{d}} b_{\varepsilon}^{g}(x)$. We note that it follows from (A.1) that

$$
\bar{b}_{\varepsilon}^{\frac{1}{\phi}} \leq c_{13} \varepsilon^{-\alpha} .
$$

LEMMA 5. If (A.1), (A.3), and (A.4) are satisfied, then there exists $c_{14}=$ $c_{14}(\phi, M, \alpha, d)$ and $c_{15}=c_{15}(\phi, \alpha, d)$ such that

$$
f_{n, \varepsilon}(x, y) \leq c_{14}\left(\bar{b}_{\varepsilon}+c_{15}\right)^{n+d / \alpha} n^{-d / \alpha}, \quad x, y \in \mathbb{R}^{d}, \varepsilon \in\left(0, \varepsilon_{0} \wedge 1\right), n \in \mathbb{N} .
$$

Proof. We may choose $n_{0} \in \mathbb{N}$ such that

$$
\left(1-c_{6} / c_{5}\right)^{n}(n+1)^{d / \alpha}<\frac{1}{n+1}
$$


for every $n \geq n_{0}$. For $n \leq n_{0}$ by Lemma 4 , we have

$$
f_{n, \varepsilon}(x, y) \leq c_{12} \bar{b}_{\varepsilon}^{d / \alpha} \bar{b}_{\varepsilon}^{n} \leq c_{12} \bar{b}_{\varepsilon}^{n+d / \alpha} n^{-d / \alpha} n_{0}^{d / \alpha}
$$

which yields the inequality (12) with $c_{14}=c_{12} n_{0}^{d / \alpha}$ in this case. For $n \geq n_{0}$, we use induction. We assume that (12) holds for some $n \geq n_{0}$ with $c_{14}=$ $\max \left(c_{12} n_{0}^{d / \alpha}, M \eta^{-\alpha-d} c_{6}{ }^{-1-d / \alpha}\right)$ and $c_{15}=\bar{b}_{1}^{\frac{1}{\phi}-1}$, where

$$
p=\frac{d 2^{\max (d / \alpha, 1)-1}}{\alpha}, \text { and } \eta=\left(\frac{c_{4}^{2} /\left(c_{13}\left(c_{5}+c_{15}\right)\right)}{2+2 p}\right)^{\frac{1}{\alpha}}
$$

We have

$$
\int f_{n, \varepsilon}(x, z) f_{\varepsilon}(z, y) \mathrm{d} z=\int_{B\left(y, \eta \varepsilon(n+1)^{1 / \alpha}\right)^{c}}+\int_{B\left(y, \eta \varepsilon(n+1)^{1 / \alpha}\right)}=I+I I .
$$

By (A.1), (A.4), and (6), we get

$$
\begin{aligned}
I & =\int_{B\left(y, \eta \varepsilon(n+1)^{1 / \alpha}\right)^{c}} f_{n, \varepsilon}(x, z) f_{\varepsilon}(z, y) \mathrm{d} z \\
& \leq M \int_{B\left(y, \eta \varepsilon(n+1)^{1 / \alpha}\right)^{c}} f_{n, \varepsilon}(x, z)|y-z|^{-\alpha-d} \mathrm{~d} z \\
& \leq M \eta^{-\alpha-d} \varepsilon^{-\alpha-d}(n+1)^{-1-d / \alpha} \int f_{n, \varepsilon}(x, z) \mathrm{d} z \\
& \leq M \eta^{-\alpha-d} c_{6}{ }^{-1-d / \alpha} \bar{b}_{\varepsilon}^{1+d / \alpha}(n+1)^{-1-d / \alpha}\left[\bar{b}_{\varepsilon}^{n}-\left(\bar{b}_{\varepsilon}-b_{\varepsilon}(x)\right)^{n}\right] .
\end{aligned}
$$

By induction, the symmetry of $f_{\varepsilon}$ and (A.4), we obtain

$$
\begin{aligned}
I I & =\int_{B\left(y, \eta \varepsilon(n+1)^{1 / \alpha}\right)} f_{n, \varepsilon}(x, z) f_{\varepsilon}(z, y) \mathrm{d} z \\
& \leq c_{14}\left(\bar{b}_{\varepsilon}+c_{15}\right)^{n+d / \alpha} n^{-d / \alpha} \int_{B\left(y, \eta \varepsilon(n+1)^{1 / \alpha}\right)} \frac{f_{\varepsilon}(y, z)}{\phi(|z-y|)} \mathrm{d} z \\
& =c_{14}\left(\bar{b}_{\varepsilon}+c_{15}\right)^{n+d / \alpha} n^{-d / \alpha}\left(b_{\varepsilon}^{\frac{1}{\phi}}(y)-b_{\eta \varepsilon(n+1)^{1 / \alpha}}^{\frac{1}{\phi}}(y)\right) \\
& \leq c_{14}\left(\bar{b}_{\varepsilon}+c_{15}\right)^{n+d / \alpha} n^{-d / \alpha} b_{\varepsilon}^{\frac{1}{\phi}}(y)\left(1-\frac{c_{4} \eta^{-\alpha}}{c_{13}(n+1)}\right) .
\end{aligned}
$$

By (13), we also have

$$
\left(1-\frac{b_{\varepsilon}(x)}{\bar{b}_{\varepsilon}}\right)^{n}(n+1)^{d / \alpha} \leq\left(1-c_{6} / c_{5}\right)^{n}(n+1)^{d / \alpha} \leq \frac{1}{n+1} .
$$


Using the fact that $\phi(a)=1$ for $a \in[0,1]$ and $b_{\varepsilon}^{\frac{1}{\phi}}(y)-b_{\varepsilon}(y)=b_{1}^{\frac{1}{\phi}-1}(y) \leq c_{15}$, we get

$$
\begin{aligned}
f_{n+1, \varepsilon}(x, y)= & I+I I+\left(\bar{b}_{\varepsilon}-b_{\varepsilon}(y)\right) f_{n, \varepsilon}(x, y)+\left(\bar{b}_{\varepsilon}-b_{\varepsilon}(x)\right)^{n} f_{\varepsilon}(x, y) \\
\leq & c_{14} \bar{b}_{\varepsilon}^{1+d / \alpha}(n+1)^{-1-d / \alpha}\left[\bar{b}_{\varepsilon}^{n}-\left(\bar{b}_{\varepsilon}-b_{\varepsilon}(x)\right)^{n}\right] \\
& +c_{14}\left(\bar{b}_{\varepsilon}+c_{15}\right)^{n+d / \alpha} n^{-d / \alpha} b_{\varepsilon}^{\frac{1}{\phi}}(y)\left(1-\frac{c_{4} \eta^{-\alpha}}{c_{13}(n+1)}\right) \\
& +c_{14}\left(\bar{b}_{\varepsilon}+c_{15}\right)^{n+d / \alpha} n^{-d / \alpha}\left(\bar{b}_{\varepsilon}-b_{\varepsilon}(y)\right) \\
& +c_{14} \bar{b}_{\varepsilon}^{1+d / \alpha}\left(\bar{b}_{\varepsilon}-b_{\varepsilon}(x)\right)^{n} \\
\leq & c_{14}\left(\bar{b}_{\varepsilon}+c_{15}\right)^{n+1+d / \alpha}(n+1)^{-d / \alpha}\left[\frac{1}{n+1}\left(1-\left(1-\frac{b_{\varepsilon}(x)}{\bar{b}_{\varepsilon}}\right)^{n}\right)\right. \\
& -\frac{b_{\varepsilon}^{\frac{1}{\phi}}(y)}{\bar{b}_{\varepsilon}+c_{15}}\left(1+\frac{1}{n}\right)^{d / \alpha} \frac{c_{4} \eta^{-\alpha}}{c_{13}(n+1)}+\left(1+\frac{1}{n}\right)^{d / \alpha} \\
& \left.+\left(1-\frac{b_{\varepsilon}(x)}{\bar{b}_{\varepsilon}}\right)^{n}(n+1)^{d / \alpha}\right] .
\end{aligned}
$$

By (A.1), (A.4), (14), and the following inequality

$$
\frac{b_{\varepsilon}^{\frac{1}{\phi}}(y)}{\bar{b}_{\varepsilon}+c_{15}} \geq \frac{c_{4} \varepsilon^{-\alpha}}{c_{5} \varepsilon^{-\alpha}+c_{15}} \geq \frac{c_{4}}{c_{5}+c_{15}},
$$

the last expression is bounded above by

$$
\begin{aligned}
& c_{14}\left(\bar{b}_{\varepsilon}+c_{15}\right)^{n+1+d / \alpha}(n+1)^{-d / \alpha} \\
& \times\left[\frac{2}{n+1}+\left(1+\frac{1}{n}\right)^{d / \alpha}\left(1-\frac{\eta^{-\alpha} c_{4}^{2} /\left(c_{13}\left(c_{5}+c_{15}\right)\right)}{n+1}\right)\right]
\end{aligned}
$$

and, finally, by the inequality

$$
\left(1+\frac{1}{n}\right)^{d / \alpha} \leq\left(1+\frac{p}{n}\right)
$$

this is smaller or equal to

$$
\begin{aligned}
& c_{14}\left(\bar{b}_{\varepsilon}+c_{15}\right)^{n+1+d / \alpha}(n+1)^{-d / \alpha} \\
& \quad \times\left[\frac{2}{n+1}+\left(1+\frac{p}{n}\right)\left(1-\frac{\eta^{-\alpha} c_{4}^{2} /\left(c_{13}\left(c_{5}+c_{15}\right)\right)}{n+1}\right)\right] \\
& \leq c_{14}\left(\bar{b}_{\varepsilon}+c_{15}\right)^{n+1+d / \alpha}(n+1)^{-d / \alpha} \\
& \quad \times\left[1-\frac{1}{n+1}\left(\eta^{-\alpha} c_{4}^{2} /\left(c_{13}\left(c_{5}+c_{15}\right)\right)-2-2 p\right)\right],
\end{aligned}
$$


which gives

$$
f_{n+1, \varepsilon}(x, y) \leq c_{14}\left(\bar{b}_{\varepsilon}+c_{15}\right)^{n+1+d / \alpha}(n+1)^{-d / \alpha} .
$$

Using the above lemmas, we may estimate $\Gamma_{\varepsilon}^{n}$ and in consequence also the exponent operator $\mathrm{e}^{t \mathcal{A}_{\varepsilon}}=\mathrm{e}^{-t \bar{b}_{\varepsilon}} \mathrm{e}^{t \Gamma_{\varepsilon}}$.

LEMMA 6. Assume (A.1)-(A.4). Then, for all $x \in \mathbb{R}^{d}$ and all nonnegative $\varphi \in$ $B_{b}\left(\mathbb{R}^{d}\right)$ such that $x \notin \operatorname{supp}(\varphi)$, we have

$$
\mathrm{e}^{t \mathcal{A}_{\varepsilon}} \varphi(x) \leq c_{10} t \exp \left(c_{11} t\right) \int \varphi(y) \frac{\phi(|y-x|)}{|y-x|^{\alpha+d}} \mathrm{~d} y, \quad \varepsilon \in(0,1) .
$$

Proof. By (7) and Lemma 3 for every $\varphi$ such that $x \notin \operatorname{supp}(\varphi)$, we get

$$
\Gamma_{\varepsilon}^{n} \varphi(x) \leq \int \varphi(y) c_{10} n\left(\bar{b}_{\varepsilon}+c_{11}\right)^{n-1} \frac{\phi(|y-x|)}{|y-x|^{\alpha+d}} \mathrm{~d} y,
$$

and

$$
\begin{aligned}
\mathrm{e}^{t \mathcal{A}_{\varepsilon}} \varphi(x) & \leq c_{10} \mathrm{e}^{-t \bar{b}_{\varepsilon}} \sum_{n=1}^{\infty} \frac{t^{n} n\left(\bar{b}_{\varepsilon}+c_{11}\right)^{n-1}}{n !} \int \varphi(y) \frac{\phi(|y-x|)}{|y-x|^{\alpha+d}} \mathrm{~d} y \\
& =c_{10} \mathrm{e}^{-t \bar{b}_{\varepsilon}} t \sum_{n=0}^{\infty} \frac{t^{n}\left(\bar{b}_{\varepsilon}+c_{11}\right)^{n}}{n !} \int \varphi(y) \frac{\phi(|y-x|)}{|y-x|^{\alpha+d}} \mathrm{~d} y \\
& =c_{10} t \exp \left(c_{11} t\right) \int \varphi(y) \frac{\phi(|y-x|)}{|y-x|^{\alpha+d}} \mathrm{~d} y .
\end{aligned}
$$

LEMMA 7. Assume (A.1), (A.3), and (A.4). Then, there is a constant $c_{16}=$ $c_{16}(\phi, M, \alpha, d)$ such that for every nonnegative $\varphi \in B_{b}\left(\mathbb{R}^{d}\right) \cap L_{1}\left(\mathbb{R}^{d}\right)$, we have

$$
\mathrm{e}^{t \mathcal{A}_{\varepsilon}} \varphi(x) \leq c_{16} \exp \left(c_{15} t\right) t^{-d / \alpha} \int \varphi(y) \mathrm{d} y+\mathrm{e}^{-t b_{\varepsilon}(x)} \varphi(x),
$$

for $x \in \mathbb{R}^{d}, \varepsilon \in\left(0, \varepsilon_{0} \wedge 1\right), t>0$.

Proof. We directly deduce from Lemma 5 that for every $\varphi \in B_{b}\left(\mathbb{R}^{d}\right) \cap L_{1}\left(\mathbb{R}^{d}\right)$

$$
\Gamma_{\varepsilon}^{n} \varphi(x) \leq c_{14}\left(\bar{b}_{\varepsilon}+c_{15}\right)^{n+d / \alpha} n^{-d / \alpha} \int \varphi(y) \mathrm{d} y+\left(\bar{b}_{\varepsilon}-b_{\varepsilon}(x)\right)^{n} \varphi(x),
$$

and, consequently, by [29, Lemma 9], we obtain

$$
\begin{aligned}
\mathrm{e}^{t \mathcal{A}_{\varepsilon}} \varphi(x) & \leq \mathrm{e}^{-t \bar{b}_{\varepsilon}}\left[c_{14} \int \varphi(y) \mathrm{d} y \sum_{n=1}^{\infty} \frac{t^{n}\left(\bar{b}_{\varepsilon}+c_{15}\right)^{n+d / \alpha}}{n ! n^{d / \alpha}}+\mathrm{e}^{t\left(\bar{b}_{\varepsilon}-b_{\varepsilon}(x)\right)} \varphi(x)\right] \\
& \leq c_{16} \exp \left(c_{15} t\right) t^{-d / \alpha} \int \varphi(y) \mathrm{d} y+\mathrm{e}^{-t b_{\varepsilon}(x)} \varphi(x) .
\end{aligned}
$$


Proof of Theorem 1. Let $t>0, \varphi \in B_{b}\left(\mathbb{R}^{d}\right)$, and $x \in \mathbb{R}^{d}$. Denote $D=$ $\left\{y \in \mathbb{R}^{d}: \phi(|y-x|)|y-x|^{-\alpha-d}<t^{-1-d / \alpha}\right\}$. Using Lemma 6 for $\mathbf{1}_{D} \varphi$ and Lemma 7 for $\mathbf{1}_{D^{c}} \varphi$, we obtain

$$
\begin{aligned}
\mathrm{e}^{t \mathcal{A}_{\varepsilon}} \varphi(x)= & \mathrm{e}^{t \mathcal{A}_{\varepsilon}}\left[\mathbf{1}_{D} \varphi\right](x)+\mathrm{e}^{t \mathcal{A}_{\varepsilon}}\left[\mathbf{1}_{D^{c}} \varphi\right](x) \\
\leq & C_{1} \mathrm{e}^{C_{2} t}\left[\int_{D} \varphi(y) \frac{t \phi(|y-x|)}{|y-x|^{\alpha+d}} \mathrm{~d} y+\int_{D^{c}} \varphi(y) t^{-d / \alpha} \mathrm{d} y\right] \\
& +\mathrm{e}^{-t b_{\varepsilon}(x)} \varphi(x) \\
\leq & C_{1} \mathrm{e}^{C_{2} t} \int \varphi(y) \min \left(t^{-d / \alpha}, \frac{t \phi(|y-x|)}{|y-x|^{\alpha+d}}\right) \mathrm{d} y+\mathrm{e}^{-t b_{\varepsilon}(x)} \varphi(x) .
\end{aligned}
$$

Proof of Theorem 2. By Lemma 12 in [29], we have

$$
\lim _{\varepsilon \rightarrow 0}\left\|\mathcal{A} \varphi-\mathcal{A}_{\varepsilon} \varphi\right\|_{\infty}=0
$$

for every $\varphi \in C_{\infty}^{2}\left(\mathbb{R}^{d}\right)$. A closure of $\mathcal{A}$ is a generator of a semigroup and from the Hille-Yosida theorem, it follows that the range of $\lambda-\mathcal{A}$ is dense in $C_{\infty}\left(\mathbb{R}^{d}\right)$ and therefore by Theorem 5.2 in [31] (see also [11]), we get

$$
\lim _{\varepsilon \downarrow 0}\left\|\mathrm{e}^{t \mathcal{A}_{\varepsilon}} \varphi-P_{t} \varphi\right\|_{\infty}=0
$$

for every $\varphi \in C_{\infty}\left(\mathbb{R}^{d}\right)$. By Theorem 1 , this yields

$$
P_{t} \varphi(x) \leq C_{1} \mathrm{e}^{C_{2} t} \int \varphi(z) \min \left(t^{-d / \alpha}, \frac{t \phi(|z-x|)}{|z-x|^{\alpha+d}}\right) \mathrm{d} z,
$$

for every nonnegative $\varphi \in C_{\infty}\left(\mathbb{R}^{d}\right)$.

\section{Discussion of examples}

We now prove the condition (A.1) (c) for functions $\phi$ of the form (2) for restricted set of parameters $\beta$ and $\gamma$. First, we recall some well-known geometric fact, see, e.g., [23, Lemma 5.3].

LEMMA 8. The volume of intersection of two balls $B(x, p+k)$ and $B(y, n-$ p) such that $|y-x|=n \in \mathbb{N}, 1 \leq p \leq n-1,0<k \leq n-p$ is less than $c k^{\frac{d+1}{2}}(\min \{p+k, n-p\})^{\frac{d-1}{2}}$.

PROPOSITION 1. Let the function $\phi$ be of the form (2). Then, the assumption (A.1) (c) is satisfied if $\beta \in(0,1]$ and $\gamma<d / 2+\alpha-1 / 2$.

Proof. Let $\beta \in(0,1]$ and $\gamma<d / 2+\alpha-1 / 2$. First, note that there is an absolute constant $C=C(m, \beta, \gamma, \alpha, d)$ such that $\phi(s) s^{-d-\alpha} \leq C \phi(u) u^{-d-\alpha}$ for $|s-u| \leq 1$ 
whenever $s, u \geq 1$. By this fact, with no loss of generality, we may and do consider only the case when $|x-y|=n$ for some even natural number $n \geq 4$. Let

$$
\begin{aligned}
\int_{B(x, 1)^{c}} \cap B(y, 1)^{c} & \frac{\phi(|y-z|)}{|y-z|^{\alpha+d}} \frac{\phi(|z-x|)}{|z-x|^{\alpha+d}} \mathrm{~d} z \\
& \leq 2 \int_{B(x, 1)^{c} \cap B(y, n-1)^{c}}+\int_{\left(B(x, 1)^{c} \cap B(x, n-1)\right) \cup\left(B(y, 1)^{c} \cap B(y, n-1)\right)} \\
& =2 I+I I .
\end{aligned}
$$

We have

$$
I \leq \frac{\phi(n-1)}{(n-1)^{\alpha+d}} \int_{B(0,1)^{c}} \frac{\phi(|z|)}{|z|^{\alpha+d}} \mathrm{~d} z \leq C \frac{\phi(|y-x|)}{|y-x|^{\alpha+d}}
$$

with some constant $C=C(m, \beta, \gamma, \alpha, d)$.

To estimate the term II, we will need the additional notations. For $1 \leq p<n / 2$ and $0 \leq k<n-p$, we denote

- $D_{p}:=\left\{z \in \mathbb{R}^{d}: n-p-1 \leq|z-y|<n-p,|x-z|<|y-z|\right\}$,

- $D_{p, k}=D_{p} \cap\left\{z \in \mathbb{R}^{d}: p+k \leq|z-x|<p+k+1\right\}$,

- $n_{p}:=\max \left\{k \in \mathbb{N}: D_{p, k} \neq \varnothing\right\}$.

Clearly, $D_{p} \subset \bigcup_{k=0}^{n_{p}} D_{p, k}$ and $D_{p, k} \subset B(x, p+k+1) \cap B(y, n-p)$. We have

$$
\begin{aligned}
I I & \leq 2^{\alpha+d-\gamma+1}|y-x|^{-\alpha-d+\gamma} \int_{1 \leq|y-z|<n-1,|x-z|<|y-z|} \frac{\mathrm{e}^{-m|x-z|^{\beta}} \mathrm{e}^{-m|y-z|^{\beta}}}{|x-z|^{\alpha+d-\gamma}} \mathrm{d} z \\
& =2^{\alpha+d-\gamma+1}|y-x|^{-\alpha-d+\gamma} \sum_{p=1}^{n / 2-1} \int_{D_{p}} \frac{\mathrm{e}^{-m|x-z|^{\beta}} \mathrm{e}^{-m|y-z|^{\beta}}}{|x-z|^{\alpha+d-\gamma}} \mathrm{d} z \\
& \leq 2^{\alpha+d-\gamma+1}|y-x|^{-\alpha-d+\gamma} \sum_{p=1}^{n / 2-1} \sum_{k=0}^{n_{p}} \int_{D_{p, k}} \frac{\mathrm{e}^{-m|x-z|^{\beta}} \mathrm{e}^{-m|y-z|^{\beta}}}{|x-z|^{\alpha+d-\gamma}} \mathrm{d} z \\
& \leq 2^{\alpha+d-\gamma+1}|y-x|^{-\alpha-d+\gamma} \sum_{p=1}^{n / 2-1} \sum_{k=0}^{n_{p}} \frac{\mathrm{e}^{-m(p+k)^{\beta}} \mathrm{e}^{-m(n-p-1)^{\beta}}}{(p+k)^{\alpha+d-\gamma}}\left|D_{p, k}\right| .
\end{aligned}
$$

Notice that $(n-p)^{\beta}-(n-p-1)^{\beta} \leq \beta$ when $\beta \in(0,1]$. Furthermore, since $p+k \leq p+n_{p}<n-p$, we also have $k^{\beta}+n^{\beta} \leq(p+k)^{\beta}+(n-p)^{\beta}$. These inequalities and Lemma 8 thus yield

$$
\begin{aligned}
I I & \leq C \frac{\mathrm{e}^{-m n^{\beta}}}{|y-x|^{\alpha+d-\gamma}} \sum_{p=1}^{n / 2-1} \sum_{k=0}^{n_{p}} \mathrm{e}^{-m k^{\beta}} k^{\frac{d+1}{2}}(p+k)^{-\alpha-d+\gamma}(p+k)^{\frac{d-1}{2}} \\
& \leq C \frac{\mathrm{e}^{-m|y-x|^{\beta}}}{|y-x|^{\alpha+d-\gamma}} \sum_{p=1}^{\infty} p^{-\frac{d+1}{2}-\alpha+\gamma} \sum_{k=0}^{\infty} \mathrm{e}^{-m k^{\beta}} k^{\frac{d+1}{2}}
\end{aligned}
$$

for some $C=C(m, \beta, \gamma, \alpha, d)$. We conclude by observing that for $\beta>0$ and $\gamma<d / 2+\alpha-1 / 2$, the last two sums are bounded by constant. 
REMARK 1. (1) When $\beta>1$, then the condition (c) in assumption (A.1) fails. This can be shown by estimating from below the integral

$$
\int_{B((x+y) / 2,1)} \frac{\phi(|y-z|)}{|y-z|^{\alpha+d}} \frac{\phi(|z-x|)}{|z-x|^{\alpha+d}} d z
$$

for $|y-x|$ big enough.

(2) Also, if $\beta=1$ and $\gamma=d / 2+\alpha-1 / 2$, then at least for $d=1$ the condition (c) in assumption (A.1) does not hold. In this case we have

$$
\int_{1}^{x-1} \mathrm{e}^{-(x-z)}(x-z)^{-1} \mathrm{e}^{-z} z^{-1} d z=2 \log (x-1) \mathrm{e}^{-x} x^{-1}, \quad x>2 .
$$

\section{Acknowledgments}

We thank the referee for careful reading of the manuscript and helpful suggestions and comments.

Open Access. This article is distributed under the terms of the Creative Commons Attribution License which permits any use, distribution, and reproduction in any medium, provided the original author(s) and the source are credited.

\section{REFERENCES}

[1] Barlow, M. T., Grigor'yan, A., Kumagai, T., Heat kernel upper bounds for jump processes and the first exit time. J. Reine Angew. Math. 626, 135-157 (2009).

[2] Blumenthal, R. M., Getoor, R. K.: Some theorems on stable processes. Trans. Amer. Math. Soc. 95, 263-273 (1960).

[3] Bogdan, K., Jakubowski, T.: Estimates of heat kernel of fractional Laplacian perturbed by gradient operators. Comm. Math. Phys. 271 (1), 179-198 (2007).

[4] Bogdan, K., Sztonyk, P.: Estimates of potential kernel and Harnack's inequality for anisotropic fractional Laplacian. Stud. Math. 181, No. 2, 101-123 (2007).

[5] Böttcher, B., Schilling, R. L.: Approximation of Feller processes by Markov chains with Lévy increments. Stoch. Dyn. 9, No. 1, 71-80 (2009).

[6] Chen, Z.-Q., Kim, P. and Kumagai, T.: Global Heat Kernel Estimates for Symmetric Jump Processes. Trans. Amer. Math. Soc., 363, no. 9, 5021-5055 (2011).

[7] Chen, Z.-Q., Kumagai, T.: Heat kernel estimates for stable-like processes on $d$-sets. Stochastic Process. Appl. 108, no. 1, 27-62 (2003).

[8] Chen, Z.-Q., Kumagai, T.: Heat kernel estimates for jump processes of mixed types on metric measure spaces. Probab. Theory Relat. Fields 140, No. 1-2, 277-317 (2008).

[9] Ethier, S. N., Kurtz, T. G.: Markov processes: characterization and convergence. Wiley Series in Probability and Mathematical Statistics, John Wiley, New York-Chicester-Brisbane-TorontoSingapore (1986).

[10] Grigor'yan A., Hu J., Lau K-S.: Comparison inequalities for heat semigroups and heat kernels on metric measure spaces, J Funct Anal 259: 2613-2641 (2010).

[11] Hasegawa, M.: A Note on the Convergence of Semi-groups of Operators. Proc. Japan Acad. 40, 262-266 (1964).

[12] Hoh, W.: Pseudo differential operators generating Markov processes. Habilitationsschrift, Universität Bielefeld 1998.

[13] Hoh, W.: The martingale problem for a class of pseudo differential operators. Math. Ann. 300, 121-147 (1994). 
[14] Hoh, W.: A symbolic calculus for pseudo differential operators generating Feller semigroups. Osaka J. Math. 35, 798-820 (1998).

[15] Jacob, N.: Feller semigroups, Dirichlet forms and pseudo differential operators. Forum Math. 4, 433-446 (1992).

[16] Jacob, N.: A class of Feller semigroups generated by pseudo differential operators. Math. Z. 215, 151-166 (1994).

[17] Jacob, N.: Pseudo differential operators and Markov processes. Vol. I : Fourier analysis and semigroups. Imperial College Press, London (2001).

[18] Jacob, N.: Pseudo-Differential Operators and Markov Processes, Vol. 2 : Generators and Their Potential Theory. Imperial College Press, London (2002).

[19] Jacob, N.: Pseudo-Differential Operators and Markov Processes, Vol. 3 : Markov Processes and Applications. Imperial College Press, London (2005).

[20] N. Jacob, V. Knopova, S. Landwehr, R. Schilling, A geometric interpretation of the transition density of a symmetric Lévy process, Sci. China Math. 55 (2012), no. 6, 1099-1126.

[21] Knopova, V., Kulik, A.: Exact asymptotic for distribution densities of Lévy functionals, Electronic Journal of Probability 16, 1394-1433 (2011).

[22] Knopova, V., Schilling, R.: Transition density estimates for a class of Lévy and Lévy-type processes, J. Theoret. Probab. 25 (1), 144-170 (2012).

[23] Kulczycki. T, Siudeja B.: Intrinsic ultracontractivity of the Feynman-Kac semigroup for the relativistic stable process, Trans. Amer. Math. Soc. 358 (11), 5025-5057 (2006).

[24] Lewandowski, M. Point regularity of $p$-stable density in $\mathcal{R}^{d}$ and Fisher information. Probab. Math. Stat. 19, No.2, 375-388 (1999).

[25] Mimica, A. Heat kernel upper estimates for symmetric jump processes with small jumps of high intensity, Potential Anal. 36, no. 2, 203-222 (2012).

[26] Schilling, R. L., Uemura, T.: On the Feller property of Dirichlet forms generated by pseudo differential operators. Tohoku Math. J. (2) 59, no. 3, 401-422 (2007).

[27] Sztonyk, P.: Regularity of harmonic functions for anisotropic fractional Laplacians. Math. Nachr. 283 No. 2, 289-311 (2010).

[28] Sztonyk, P.: Estimates of tempered stable densities, J. Theoret. Probab. 23 (1), 127-147 (2010).

[29] Sztonyk, P.: Approximation of Stable-dominated Semigroups, Potential Anal. 33, 211-226 (2010).

[30] Sztonyk, P.: Transition density estimates for jump Lévy processes, Stochastic Process. Appl. 121, 1245-1265 (2011).

[31] Trotter, H. F.: Approximation of semi-groups of operators. Pacific J. Math. 8 (1958), 887-919.

[32] Watanabe, T.: Asymptotic estimates of multi-dimensional stable densities and their applications. Trans. Am. Math. Soc. 359, No. 6, 2851-2879 (2007).

\author{
Kamil Kaleta \\ Institute of Mathematics, \\ University of Warsaw, \\ ul. Banacha 2, \\ 02-097 Warszawa, Poland. \\ Kamil Kaleta and Pawet Sztonyk \\ Institute of Mathematics and Computer \\ Science, \\ Wroctaw University of Technology, \\ Wybrzeże Wyspiańskiego 27, \\ 50-370 Wroctaw, Poland. \\ E-mail:Kamil.Kaleta@pwr.wroc.pl \\ Pawet Sztonyk \\ E-mail:Pawel.Sztonyk@pwr.wroc.pl
}

